\title{
CONHECIMENTOS E PRÁTICAS SOBRE FATORES DE RISCO PARA DOENÇAS CRÔNICAS NÃO TRANSMISSIVEES, EM IDOSOS DE UM BAIRRO DE FOZ DO IGUACUU, PARANÁ, ADSCRITOS À ESTRATÉETA SAÚDE DA FAMILIA
}

\section{Knowledge and practices regarding risk factors for ehronic diseases in the elderly in a neighhorhood of Foz do Iguaçu, Paraná, enrolled in the Family Health Strategy}

\author{
Loren Milagros Salazar Cardoza ${ }^{1}$, Andreia Pereira dos Santos ${ }^{2}$, \\ Alessandra Cristiane Sibim ${ }^{3}$, Carmen Justina Gamarra ${ }^{4}$
}

\section{RESUMO}

O impacto das doenças e agravos não transmissíveis sobre as sociedades humanas é crescente. As principais doenças deste grupo são as do aparelho circulatório, câncer, respiratórias crônicas, diabetes e musculoesqueléticas. São doenças multifatoriais relacionadas a fatores de riscos modificáveis, como o tabagismo, o consumo excessivo de bebidas alcoólicas, a obesidade, as dislipidemias, o consumo excessivo de sal, a ingestão insuficiente de frutas e verduras e a inatividade física. As doenças não transmissíveis incidem sobre a população adulta e, particularmente, sobre os idosos de modo mais intenso, trazendo consequências negativas para a qualidade de vida desse grupo e provocando maiores gastos com assistência hospitalar em saúde. Este estudo teve como principal objetivo avaliar os conhecimentos e práticas sobre fatores de riscos para doenças crônicas não transmissíveis, numa população idosa de um bairro de Foz do Iguaçu, Paraná, adscrita à estratégia Saúde da Família. Realizou-se um inquérito domiciliar com pessoas de 60 anos de idade ou mais residentes no bairro selecionado. A partir da listagem de idosos, selecionou-se uma amostra aleatória simples. Os dados foram digitados e analisados por meio do programa Epi Info 7.0, sendo submetidos a técnicas estatísticas exploratórias e de análise de associação. O conhecimento sobre os fatores de risco observado neste estudo é satisfatório (variando de $46.43 \%$ a $78.57 \%$ ); porém, como tem sido discutido na literatura, o conhecimento nem sempre implica em uma prática efetiva, o que também é possível observar neste estudo. A prática adequada de alimentação, consumo de álcool, atividade física e tabagismo, no cotidiano das pessoas, vai além de ter ou não conhecimento. É preciso pensar nas ações de promoção da saúde, levando em conta

\section{ABSTRACT}

The impact of non-infectious diseases and disorders on human societies is increasing. The main diseases in this group are those of the circulatory system, cancer, chronic respiratory, diabetes, and musculoskeletal. These are multifactorial diseases related to modifiable risk factors such as smoking, excessive alcohol consumption, obesity, dyslipidemia, excessive salt intake, inadequate intake of fruit and vegetables, and physical inactivity. Non-infectious diseases afflict the adult population and particularly the elderly in a most intense way, producing negative consequences for the quality of life of this group and leading to higher spending on hospital health care. This study aimed to evaluate the knowledge and practices regarding risk factors for chronic non-infectious diseases in an elderly population of a neighborhood of Foz do Iguaçu, Paraná, enrolled in the Family Health Strategy. We conducted a household survey with people age 60 years or older living in that neighborhood. From this list of elderly persons, a simple random sample was selected. Data were entered and analyzed using the Epi Info 7.0 program, and analyzed using exploratory statistical techniques and association analysis. Knowledge of the risk factors observed in this study is satisfactory (ranging from $46.43 \%$ to $78.57 \%$ ); however, as has been discussed in the literature, knowledge does not always imply an effective practice, which can likewise be observed in this study. Proper eating habits, alcohol consumption, physical activity, and smoking, as part of people's daily lives goes beyond whether or not they have knowledge. It is essential to think about health promotion actions taking into account the characteristics of the elderly population and of the national reality to thus try to ensure a genuine

\footnotetext{
Discente do Curso de Saúde Coletiva da Universidade Federal da Integração Latino-Americana (UNILA ). E-mail: loren.cardoza@aluno.unila.edu.br.

Universidade Federal da Integração Latino-Americana (UNILA).

Universidade Federal da Integração Latino-Americana (UNILA).

Universidade Federal da Integração Latino-Americana (UNILA).
} 
as características da população idosa e as características da realidade nacional, para, assim, tentar garantir o entendimento de fato das informações a serem trabalhadas e a apropriação do conhecimento.

PALAVRAS-CHAVE: Conhecimento; Fatores de Risco; Idoso; Doença Crônica.

\section{INTRODUÇÃO}

O impacto das doenças e agravos não transmissíveis sobre as sociedades humanas é crescente. Os custos econômicos e sociais delas decorrentes são grandes, seja devido à morte prematura ou incapacitação definitiva de pessoas em idade produtiva, ou ainda pela sobrecarga na demanda por serviços assistenciais. ${ }^{1}$ Trata-se de um crescente problema de saúde global e uma ameaça à saúde e ao desenvolvimento humano.

As principais doenças crônicas são as do aparelho circulatório, câncer, respiratórias crônicas, diabetes e musculoesqueléticas. São doenças multifatoriais relacionadas a fatores de riscos modificáveis, como o tabagismo, o consumo excessivo de bebidas alcoólicas, a obesidade, as dislipidemias, o consumo excessivo de sal, a ingestão insuficiente de frutas e verduras e a inatividade física. ${ }^{2}$ As diferenças no acesso aos bens e aos serviços, a baixa escolaridade, renda e as desigualdades no acesso à informação são ainda fatores determinantes para essas doenças. ${ }^{2,3}$

Estimativas da Organização Mundial da Saúde (OMS) apontam que as doenças crônicas são as principais causas de morte no mundo, às quais foram atribuídas 38 milhões de óbitos em 2012, quase $68 \%$ da mortalidade mundial. Segundo as estimativas da OMS, se essa tendência for mantida, elas deverão responder por $73 \%$ dos óbitos e $60 \%$ da carga de doenças, no ano 2020. A carga das doenças crônicas recai especialmente sobre países de baixa e média renda, o que se tornará um sério problema de saúde pública, agravando as iniquidades. Seguindo a tendência mundial, no Brasil, as doenças crônicas não transmissíveis são a causa de $74 \%$ das mortes. ${ }^{2}$

As doenças não transmissíveis incidem sobre a população adulta e, particularmente, sobre os idosos de modo mais intenso, trazendo consequências negativas para a qualidade de vida dessas populações e provocando maiores gastos com assistência hospitalar em saúde, ${ }^{1}$ pois, como sabemos, a saúde da população tem alcançado um enorme progresso mundial: a mortalidade em crianças menores de 5 anos vem diminuindo, o controle das doen- understanding of the information to be handled and the appropriation of knowledge.

KEYWORDS: Knowledge; Risk Factors; Aged; Chronic Disease.

ças infecciosas é uma realidade cada vez mais crescente e, inclusive, em algumas partes do mundo, tem havido um progresso substancial na prevenção de mortes prematuras por doenças cardíacas e câncer, desse modo, a proporção de mortes fica concentrada nos grupos de pessoas com 70 anos de idade ou mais. ${ }^{4}$ Segundo Horton ${ }^{4}$, esta transformação é determinada pela evolução social, econômica, científica e tecnológica que dissemina possibilidades de acesso ao consumo de produtos, informações e bens, antes restritos a pequenos grupos. Dessa forma, o desafio da Saúde Pública não deve se limitar à ampliação do tempo de vida, devendo propiciar também a melhoria da qualidade de vida dessa parcela da população.

O aumento da expectativa de vida traz os efeitos associados ao envelhecimento em si e o maior tempo para o surgimento e a combinação de várias doenças e para evitar altos índices de morbidade ou mortalidade por doenças crônicas de uma forma economicamente sustentável, a tendência mundial na atenção à saúde tem sido a priorização da prevenção e a promoção da saúde, com intervenções, que foquem os fatores de riscos associados a essas doenças. ${ }^{5}$ Nesse sentido, na tentativa de contribuir para a adequação das atividades de controle dessas doenças, este estudo teve como objetivo avaliar os conhecimentos e práticas sobre fatores de riscos para doenças crônicas não transmissíveis, numa população idosa de um bairro de Foz do Iguaçu, Paraná, adscrita à estratégia Saúde da Família.

\section{METODOLOGIA}

O estudo foi realizado no bairro Vila C Velha adscrito à Estratégia de Saúde da Família, no município de Foz do Iguaçu, localizado no estado do Paraná, durante janeiro a maio de 2014. Trata-se de um inquérito domiciliar com pessoas de 60 anos de idade ou mais residentes no bairro selecionado. A partir da listagem de idosos, atualizada pelos agentes comunitários da saúde da área, em agosto de 2013, selecionou-se uma amostra aleatória simples dos mesmos. 
O tamanho da amostra calculado, com base no total de 285 idosos residentes no bairro no momento do início das entrevistas, foi de 167. Foram excluídos os idosos com problemas mentais, surdez e aqueles que não se encontravam em casa após a terceira visita.

Os questionários foram aplicados por duas das pesquisadoras envolvidas no estudo, nos domicílios dos participantes. Os participantes responderam às perguntas após leitura e assinatura do termo de consentimento livre e esclarecido.

Foi elaborado um formulário estruturado, a partir de questões utilizadas em outras pesquisas ${ }^{6,7} \mathrm{com}$ perguntas sobre dados pessoais, estado de saúde, prática de hábitos saudáveis e conhecimento sobre os fatores de risco para doenças crônicas.

As variáveis estudadas foram sexo, idade (categorizada em quinquênios), escolaridade (categorizada em três tipos: nunca foi para a escola, ensino fundamental completo e ensino fundamental incompleto) renda, práticas de hábitos saudáveis e conhecimento. A renda ou renda familiar mensal foi estratificada em 3 tipos, segundo a resposta do entrevistado, o mesmo era questionado sobre a quantidade de salários mínimos por mês dos integrantes da família. Constituindo, dessa forma, o tipo 1 as rendas com valores acima de 2 salários mínimos; o tipo 2, rendas com valores entre 1-2 salários mínimos e o tipo 3, valores inferiores a 724,00 reais, referentes ao salário mínimo no início da entrevista, de acordo com a vigência do decreto n8.166, de 23 de dezembro de 2013.

A prática de hábitos saudáveis foi avaliada para cada fator de risco estudado e categorizada em adequado ou inadequado, de acordo com as respostas dos entrevistados, a saber: prática adequada para fumo, o fato de não fumar no momento da entrevista, sendo que aqueles que referiram fumar frequentemente pelo menos um cigarro por dia foram agrupados na categoria de prática inadequada para a variável fumo; em relação a sedentarismo, aqueles que referiram realizar pelos menos uma atividade física semanal como caminhada e frequentar academias públicas foram considerados não sedentários; considerouse prática adequada de consumo de álcool aqueles que referiram não beber no momento da entrevista, e aqueles que referiram beber esporadicamente (quase nunca) e, finalmente, a prática adequada de alimentação se estimou por meio do consumo regular de frutas e verduras autorreferido pelos participantes.

Para avaliar o conhecimento da relação entre fatores risco e doenças crônicas, foi investigada a associação entre cada um dos fatores de risco (sedentarismo, tabagismo, consumo excessivo de álcool e alimentação inadequada) com oito doenças crônicas (diabetes, hipertensão arterial, AIDS, osteoporose, câncer de pulmão, depressão, cirrose hepática e infarto agudo do miocárdio); sendo construído um escore de acertos e erros, que poderia variar de zero a oito pontos, os quais foram posteriormente dicotomizados em conhecimento adequado (escore maior ou igual a 5) e inadequado. A definição de certo ou errado para cada resposta baseou-se no estudo do Borges. ${ }^{7}$

Os dados foram digitados e analisados por meio do programa Epi Info 7.0, sendo submetidos a técnicas estatísticas exploratórias e de análise de associação, por meio de média, desvio padrão e razão de prevalências, respectivamente.

O projeto do estudo foi avaliado e aprovado (Número do Parecer: 475.925) pelo Comitê de Ética em Pesquisas do Conselho Nacional de Saúde/Ministério de Saúde.

\section{RESULTADOS}

Tabela 1 - Porcentagem segundo características socioeconómicas da população idosa do bairro de Vila C Velha, no município de Foz do Iguaçu, avaliadas no ano de 2014.

\begin{tabular}{|c|c|c|}
\hline Características & $\mathrm{n}^{*}$ & $\%$ \\
\hline
\end{tabular}

\begin{tabular}{|c|c|c|}
\hline Características & $\mathrm{n}^{*}$ & $\%$ \\
\hline
\end{tabular}

$\begin{array}{lcc}\text { Sexo } & & \\ \text { Feminino } & 92 & 55.09 \\ \text { Masculino } & 75 & 44.91 \\ \text { Total } & 167 & 100\end{array}$

\section{Idade}

$60+65$

$65+70$

$62 \quad 37.13$

$70+75$

$39 \quad 23.35$

75 ou mais

$38 \quad 22.75$

Total

$\begin{array}{ll}28 & 16.77\end{array}$

$167 \quad 100$ 


\begin{tabular}{lll}
\hline Características & $\mathrm{n} *$ & $\%$ \\
\hline
\end{tabular}

\section{Escolaridade}

Nunca foi para a escola

Ensino Fundamental incompleto

Ensino completo ou mais

Total

\section{Renda (em reais)}

Tipo 3

Tipo 2

Tipo 1

Total

\section{Como avaliam sua saúde}

$\begin{array}{lcc}\text { Muito boa } & 2 & 1.2 \\ \text { Boa } & 53 & 31.74 \\ \text { Regular } & 81 & 48.5 \\ \text { Ruim } & 25 & 14.97 \\ \text { Muito ruim } & 6 & 3.59 \\ \text { Total } & 167 & 100\end{array}$

\section{Estado Civil}

$\begin{array}{cclcc}31 & 18.56 & \text { Casado(a) ou mora com companheiro(a) } & 115 & 69.28 \\ 119 & 71.26 & \text { Outros estados civis } & 51 & 30.72 \\ 17 & 10.18 & \text { Total } & 166 & 100\end{array}$

$167 \quad 100$

\section{Tem algum problema de saúde}

$\begin{array}{ccllcc}42 & 25.15 & \text { Não } & 18 & 10.78 \\ 88 & 52.69 & \text { Sim } & 149 & 89.22 \\ 37 & 22.16 & \text { Total } & 167 & 100 \\ 167 & 100 & & & \end{array}$

\footnotetext{
* Diferenças nos totais devem-se a perdas de informação para algumas variáveis.
}

Fonte: dados da pesquisa.

Na Tabela 1, evidenciou-se que mais de 50\% dos participantes foram do sexo feminino, a idade predominante dos idosos foi a faixa dos $60-65$ anos. No tocante à escolaridade $71,26 \%$ possuía ensino fundamental incompleto e aproximadamente $20 \%$ referiram que nunca foram à escola. Quase 70\% dos participantes encontravam-se casados ou morando com algum companheiro no momento da pesquisa. A renda da maioria dos participantes (52,69\%) está na faixa de 724 a 1448 reais por mês.

No que se refere à saúde, quase $90 \%$ dos participantes portam algum problema de saúde, porém $48,50 \%$ avalia a sua saúde como regular, seguida de $31,74 \%$ que a avalia como boa. 
Tabela 2 - Avaliação da adequação do conhecimento sobre os fatores de risco para doenças crônicas em idosos da Vila C Velha, do município de Foz do Iguaçu avaliada no ano de 2014.

\begin{tabular}{|c|c|c|c|c|c|c|c|c|c|}
\hline \multirow{3}{*}{ Características } & \multirow[b]{3}{*}{ Total } & \multicolumn{7}{|c|}{ Conhecimento adequado } & \\
\hline & & \multicolumn{4}{|c|}{ Alimentação inadequada } & \multicolumn{4}{|c|}{ Bebida alcoólica } \\
\hline & & $\mathbf{N}$ & $(\%)$ & $\mathbf{R R}$ & $\mathbf{P}$ & $\mathbf{N}$ & $(\%)$ & $\mathbf{R R}$ & $\mathrm{p}$ \\
\hline \multicolumn{10}{|l|}{ Sexo } \\
\hline Masculino & 75 & 50 & 66.67 & -- & -- & 58 & 77.33 & -- & -- \\
\hline Feminino & 92 & 50 & 54.35 & 1.37 & $0,927-2,023$ & 62 & 67.39 & 1.44 & $0,863-2,398$ \\
\hline \multicolumn{10}{|l|}{ Idade } \\
\hline$>60-65$ & 62 & 40 & 64.52 & -- & -- & 43 & 69.35 & -- & -- \\
\hline$>65-70$ & 39 & 24 & 61.54 & 1.08 & $0,645-1,823$ & 26 & 66.67 & 1.09 & $0,609-1,944$ \\
\hline$>70-75$ & 38 & 19 & 50 & 1.41 & $0,888-2,237$ & 29 & 76.32 & 0.77 & $0,390-1,529$ \\
\hline$>75$ & 28 & 17 & 60.71 & 1.1 & $0,626-1,957$ & 22 & 78.57 & 0.7 & $0,313-1,559$ \\
\hline \multicolumn{10}{|l|}{ Renda } \\
\hline Tipo 1 & 37 & 23 & 62.16 & -- & -- & 20 & 54.05 & -- & -- \\
\hline Tipo 2 & 88 & 52 & 59.09 & 1.08 & $0,667-1,753$ & 67 & 76.14 & 0.52 & $0,311-0,866$ \\
\hline Tipo 3 & 42 & 25 & 59.52 & 1.07 & $0,616-1,859$ & 33 & 78.57 & 0.47 & $0,237-0,917$ \\
\hline \multicolumn{10}{|l|}{ Escolaridade } \\
\hline Ensino completo ou mais & 17 & 12 & 70.59 & -- & -- & 10 & 74.19 & -- & -- \\
\hline Ensino Fundamental incompleto & 119 & 71 & 59.66 & 1.37 & $0,636-2,957$ & 87 & 73.11 & 0.65 & $0,344-1,239$ \\
\hline Nunca foi para a escola & 31 & 17 & 54.84 & 1.54 & $0,668-3,529$ & 23 & 58.82 & 0.63 & $0,275-1,429$ \\
\hline \multicolumn{10}{|l|}{ Estado Civil } \\
\hline $\begin{array}{l}\text { Casado(a) ou mora com compa- } \\
\text { nheiro (a) }\end{array}$ & 115 & 71 & 61.74 & -- & -- & 86 & 74.78 & -- & -- \\
\hline Outros estados civis & 10 & 5 & 50 & 1.3 & $0,674-2,533$ & 7 & 70 & 1.18 & $0,439-3,226$ \\
\hline \multicolumn{10}{|l|}{ Tem algum problema de saúde } \\
\hline Sim & 149 & 87 & 58.39 & -- & -- & 108 & 72.48 & -- & -- \\
\hline Não & 18 & 13 & 72.22 & 0.67 & $0,309-1,440$ & 12 & 66.67 & 1.21 & $0,599-2,448$ \\
\hline
\end{tabular}




\section{Conhecimento adequado}

Características

\section{Sedentarismo}

Fumo

\begin{tabular}{lllllllll} 
Total & $\mathbf{N}$ & $(\%)$ & $\mathrm{RR}$ & $\mathbf{P}$ & $\mathbf{N}$ & $(\%)$ & $\mathrm{RR}$ & $\mathrm{p}$ \\
\hline
\end{tabular}

\section{Sexo}

$\begin{array}{lcccccccccc}\text { Masculino } & 75 & 52 & 69.33 & -- & -- & 50 & 66.67 & -- & -- \\ \text { Feminino } & 92 & 66 & 71.74 & 0.92 & 0,575-1,476 & 49 & 53.26 & 1.4 & 0,951-2,066 \\ \text { Idade } & & & & & & & & & & \\ >60-65 & 62 & 47 & 75.8 & -- & -- & 40 & 64.52 & -- & -- \\ >65-70 & 39 & 28 & 71.8 & 1.16 & 0,598-2,271 & 24 & 61.53 & 1.08 & 0,645-1,823 \\ >70-75 & 38 & 26 & 68.42 & 1.3 & 0,686-2,482 & 22 & 57.9 & 1.19 & 0,719-1,959 \\ >75 & 28 & 17 & 60.71 & 1.62 & 0,859-3,071 & 13 & 46.43 & 1.51 & 0,933-2,443\end{array}$

\section{Renda}

Tipo 1

Tipo 2

Tipo 3

$68 \quad 64$

$\begin{array}{lllllllll}42 & 28 & 66.66 & 1 & 0,539-1,852 & 23 & 54.76 & 1.05 & 0,636-1,719\end{array}$

\section{Escolaridade}

Ensino completo ou mais

$17 \quad 13 \quad 76.47$

Ensino Fundamental incompleto

$119 \quad 85$

Nunca foi para a escola

$31 \quad 20$

71.43

1.21

$0,467-1,432$

11

64.7

$64.53 \quad 1.54$

0,539-1,852

70
23

$58.83 \quad 1.17$

$0,591-2,999$

\section{Estado Civil}

Casado(a) ou mora com companheiro (a)

$\begin{array}{cccccccccc}115 & 88 & 76.52 & -- & -- & 74 & 64.35 & -- & -- \\ 10 & 6 & 60 & 1.7 & 0,745-3,898 & 6 & 60 & 1.12 & 0,505-2,492 \\ 149 & 104 & 69.79 & -- & -- & 86 & 57.72 & -- & -- \\ 18 & 14 & 77.78 & 0.74 & 0,299-1,806 & 13 & 72.22 & 0.66 & 0,305-1,416\end{array}$

Outros estados civis

Tem algum problema de saúde

$\operatorname{Sim}$

Não

Fonte: dados da pesquisa.

$\mathrm{Na}$ Tabela 2 constata que a porcentagem de conhecimento adequado para os fatores de risco, segundo as variáveis socioeconômicas, é maior que $50 \%$ para todos os fatores, à exceção do fumo em relação à idade maior que 75 anos, pois a porcentagem de conhecimento adequado nesta faixa foi de $46,43 \%$. 
Tabela 3 - Razão de prevalência de alimentação inadequada, consumo de álcool, sedentarismo e tabagismo, segundo variáveis socioeconômicas em idosos do bairro de Vila C Velha, do município de Foz do Iguaçu avaliados no ano de 2014.

\begin{tabular}{|c|c|c|c|c|c|c|c|}
\hline \multirow{2}{*}{ Variáveis } & \multirow[b]{2}{*}{ Total } & \multicolumn{3}{|c|}{ Alimentação inadequada } & \multicolumn{3}{|c|}{ Consumo de álcool } \\
\hline & & $\%$ & $\mathbf{R R}$ & $\mathrm{p}$ & $\%$ & $\mathbf{R R}$ & $\mathbf{P}$ \\
\hline \multicolumn{8}{|l|}{ Sexo } \\
\hline Masculino & 75 & 54.7 & -- & -- & 38.7 & -- & -- \\
\hline Feminino & 92 & 77.2 & 1.41 & $1,117-1,784$ & 25 & 0.64 & $0,410-1,018$ \\
\hline \multicolumn{8}{|l|}{ Idade (anos) } \\
\hline$>60-65$ & 62 & 74.2 & -- & -- & 29 & -- & -- \\
\hline$>65-70$ & 39 & 66.7 & 0.9 & $0,689-1,173$ & 28.2 & 0.97 & $0,515-1,831$ \\
\hline$>70-75$ & 38 & 55.3 & 0.74 & $0,540-1,027$ & 31.6 & 1.09 & $0,591-1,999$ \\
\hline$>75$ & 28 & 67.9 & 0.91 & $0,681-1,227$ & 39.3 & 1.35 & $0,740-2,472$ \\
\hline \multicolumn{8}{|l|}{ Renda } \\
\hline Tipo 1 & 37 & 67.6 & -- & -- & 18.9 & -- & -- \\
\hline Tipo 2 & 88 & 69.3 & 1.03 & $0,789-1,335$ & 34.1 & 1.8 & $0,870-3,730$ \\
\hline Tipo 3 & 42 & 61.9 & 0.92 & $0,661-1,269$ & 35.7 & 1.88 & $0,864-4,121$ \\
\hline \multicolumn{8}{|l|}{ Escolaridade } \\
\hline Ensino completo ou mais & 17 & 82.4 & -- & -- & 35.3 & -- & -- \\
\hline Ensino Fundamental incompleto & 119 & 69.8 & 0.9 & $0,682-1,179$ & 29.4 & 0.83 & $0,413-1,680$ \\
\hline Nunca foi para a escola & 31 & 48.4 & 0.62 & $0,401-0,965$ & 35.5 & 1 & $0,452-2,237$ \\
\hline \multicolumn{8}{|l|}{ Estado Civil } \\
\hline Casado(a) ou mora com companheiro(a) & 115 & 64.35 & -- & -- & 33.91 & -- & -- \\
\hline Outros estados civis & 10 & 70 & 1.09 & $0,709-1,669$ & 40 & 1.18 & $0,529-2,627$ \\
\hline \multicolumn{8}{|l|}{ Tem algum problema de saúde } \\
\hline $\operatorname{Sim}$ & 149 & 67.8 & -- & -- & 33.7 & -- & -- \\
\hline Não & 18 & 61.1 & 0.9 & $0,613-1,325$ & 11.1 & 0.33 & $0,088-1,247$ \\
\hline
\end{tabular}




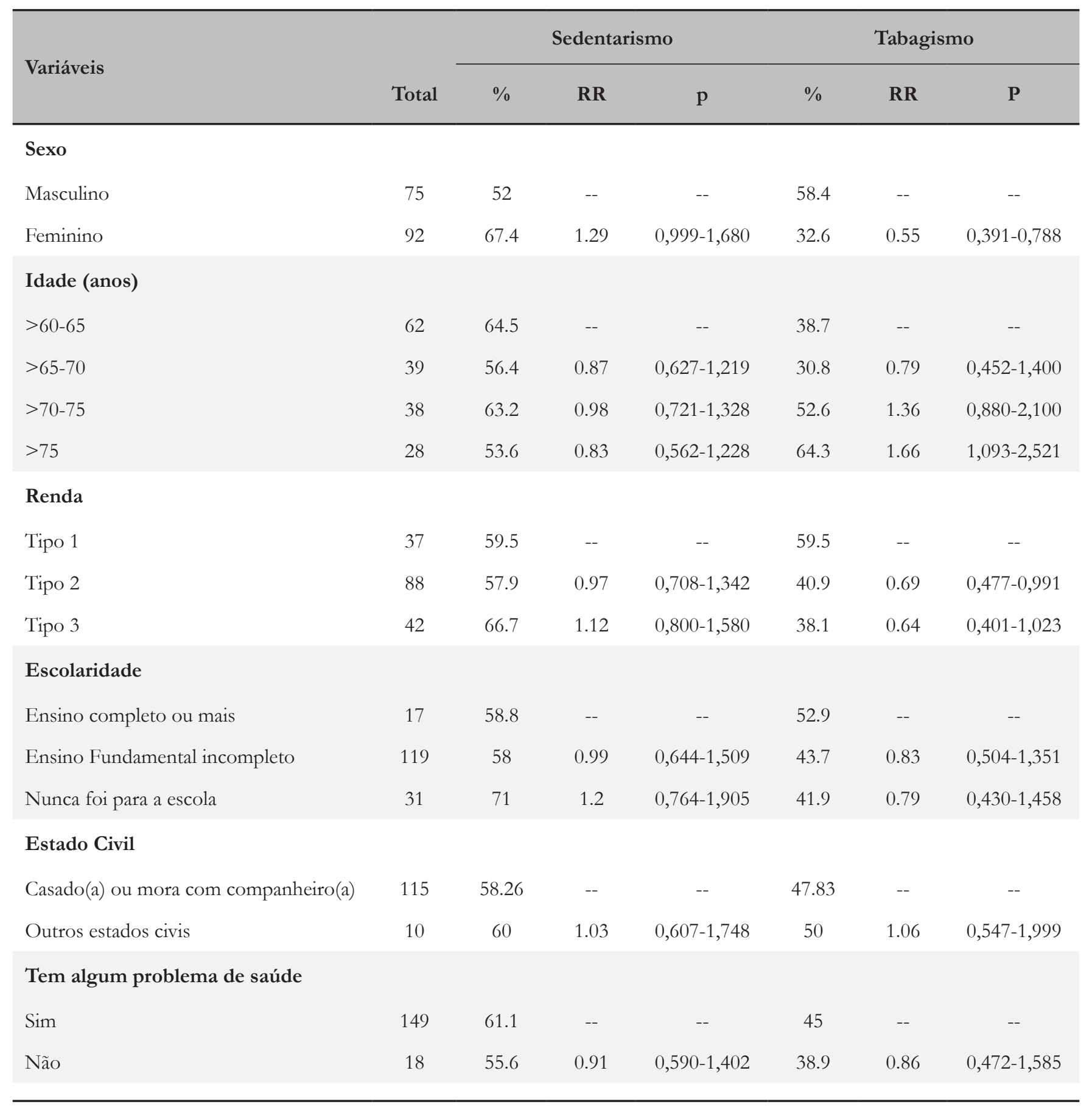

Fonte: dados da pesquisa.

Quanto à prevalência de alimentação inadequada e sedentarismo, a Tabela 3 mostra uma associação entre o sexo e essas práticas, pois as mulheres apresentam maior razão de prevalência na prática de alimentação inadequada $(77,2 \%)$ e de sedentarismo $(67,4 \%)$ que os homens, porém em relação ao tabagismo e ao consumo de álcool são os homens os que apresentam maior razão de prevalência com $58,4 \%$ e $38,7 \%$, respectivamente. Com respeito à relação de prevalência de tabagismo e a idade, esta vai aumentando progressivamente do grupo mais jovem em comparação ao mais velho. 
Tabela 4 - Prática favorável de alimentação, consumo de bebida alcoólica, atividade física e tabagismo em relação ao conhecimento adequado ou inadequado entre idosos do bairro Vila C Velha, do município de Foz do Iguaçu avaliados no ano de 2014.

\begin{tabular}{|c|c|c|c|c|c|c|c|c|}
\hline \multirow{3}{*}{ Conhecimento } & \multicolumn{8}{|c|}{ Prática Favorável } \\
\hline & \multicolumn{4}{|c|}{ Alimentação } & \multicolumn{4}{|c|}{ Bebida Alcoólica } \\
\hline & $\mathrm{n}$ & $\%$ & $\mathbf{R} \mathbf{R}$ & $\mathrm{p}$ & $\mathrm{n}$ & $\%$ & $\mathbf{R R}$ & $\mathrm{p}$ \\
\hline Adequado & 38 & 22.76 & -- & -- & 49 & 29.34 & -- & -- \\
\hline Inadequado & 65 & 38.92 & 1.14 & $0.78-1.69$ & 66 & 39.52 & 0.70 & $0.42-1.15$ \\
\hline Total & 103 & 61.68 & -- & -- & 115 & 68.86 & -- & -- \\
\hline \multirow{3}{*}{ Conhecimento } & \multicolumn{8}{|c|}{ Prática Favorável } \\
\hline & \multicolumn{4}{|c|}{ Sedentarismo } & \multicolumn{4}{|c|}{ Fumo } \\
\hline & $\mathbf{n}$ & $\%$ & $\mathbf{R} \mathbf{R}$ & $\mathrm{p}$ & $\mathbf{n}$ & $\%$ & $\mathbf{R} \mathbf{R}$ & $\mathrm{p}$ \\
\hline Adequado & 28 & 16.77 & -- & -- & 36 & 21.56 & -- & -- \\
\hline Inadequado & 38 & 22.75 & 0.91 & $0.70-1.18$ & 57 & 34.13 & 1.01 & $0.71-1.43$ \\
\hline Total & 66 & 39.52 & -- & -- & 93 & 55.69 & -- & -- \\
\hline
\end{tabular}

Fonte: dados da pesquisa.

Sobre a prática favorável de alimentação, consumo de bebida alcoólica e fumo, as porcentagens superam os $50 \%$, situação diferente à prática de atividade física, na qual só 39,5\% dos participantes da pesquisa apresentam índices favoráveis ou satisfatórios.

Do total de pessoas que exercem práticas favoráveis, de acordo com cada fator de risco estudado, menos da metade possui um conhecimento adequado.

\section{DISCUSSÃO}

Os resultados deste estudo mostram maior proporção de mulheres. No Brasil, o número de mulheres idosas é maior que o número de idosos, segundo o último censo ${ }^{8} \mathrm{e}$ estas constituem um grupo mais vulnerável, quando comparadas aos homens na mesma faixa etária. As mulheres têm maior tempo de exposição às doenças crônicas, maior tempo de atividades domésticas e tendência a viver sozinhas no final da vida,${ }^{10}$ convivendo por mais tempo com doenças. ${ }^{9}$

Neste estudo, 93,48\% das mulheres referiram ter alguma enfermidade, proporção maior à observada entre homens
(84\%). Verificou- se o mesmo evento na porcentagem de mulheres que possuem hipertensão arterial, diabetes, doença cardíaca e derrame com proporções sempre maiores no grupo de mulheres. Estes dados reforçam os achados de autores anteriormente citados. Por outro lado, as mulheres deste estudo mostraram menores escores de conhecimento para três dos quatro fatores de risco estudados, alimentação inadequada, álcool e fumo, sendo que para o fator de risco sedentarismo se mostram mais conhecedoras. Porém, em relação à prática de atividade física se mostram mais sedentárias. Os resultados do conhecimento nas mulheres poderiam ser entendidos como uma questão de gênero, principalmente pela idade das participantes.

Não obstante às mudanças no mercado de trabalho e o maior acesso à educação, as mulheres ainda possuem acesso mais restrito ${ }^{11}$ ao trabalho e educação, por distintos fatores como os socioculturais. Isso se vê refletido na ocupação ao longo da vida das mulheres participantes, sendo que apenas $21.74 \%$ referiram algum tipo de trabalho diferente das atividades próprias das donas de casa. Cabe considerar que foi analisado o conhecimento sobre diversos fatores de risco com relação a doenças específicas, o que 
implica um conhecimento mais apurado sobre a relação do fator de risco e as doenças que causam.

A idade predominante nos idosos (60-65 anos) foi relatada em outros estudos ${ }^{11,12}$ e está considerada na faixa (60-69 anos). Essa faixa apresenta mais possibilidades de desenvolver ações de promoção na saúde,,$^{13}$ pois conserva maior autonomia comparada aos grupos mais idosos. Deve-se então, aproveitar esta predominância de faixa etária para se investir na implementação das políticas públicas para idosos brasileiros.

Em relação à renda, os escores médios de conhecimento foram maiores para três dos quatro fatores de risco avaliados (alimentação inadequada, sedentarismo e fumo), nos participantes com renda tipo 1(renda maior) e, para o fator de risco álcool, o escore foi maior nas pessoas com renda de tipo 3 (renda menor). Isso pode estar atrelado ao acesso à informação, mais do que diretamente à renda, devido ao fato de os escores, de forma geral, não apresentarem diferenças muito elevadas, os meios de comunicação e a difusão em campanhas poderiam ter um papel relevante nesse aspecto. Quanto ao estado civil dos idosos, os casados ou que moravam com companheiro apresentaram escores maiores de conhecimento nos quatro fatores de risco, o que pode ser associado à maior concentração de apoio social vivenciado nesse grupo. ${ }^{23,24}$

O conhecimento sobre os fatores de risco observado neste estudo é satisfatório (variando de $46.43 \%$ a $78.57 \%$ ), acontecimento também referido em outros estudos sobre fatores de risco, ${ }^{14,15,16,17}$ o que pode ser explicado pelo trabalho educacional de promoção e prevenção realizado em diversas campanhas de saúde, a divulgação de informações na mídia, em feiras e outros, assim como a informação recebida em consultas e atividades da unidade de saúde, de acordo com as recomendações da OMS. ${ }^{18}$ Estudos futuros poderiam pesquisar as fontes da informação com o intuito de potenciar o uso dos meios mais citados.

Como tem sido discutido na literatura, ${ }^{16,19,20,21}$ o conhecimento nem sempre implica uma prática efetiva, o que também é possível observar no presente estudo. A prática adequada de alimentação, o consumo de álcool, atividade física e tabagismo no cotidiano das pessoas vão além de ter ou não conhecimento. Vale ressaltar dois resultados deste trabalho, sendo o primeiro, que a prevalência de tabagismo foi menor no grupo mais velho $(64,3 \%)$, quando comparada com o grupo mais jovem $(38,7 \%)$, resultados também relatados em outro estudo, em que se atribui à diminuição do tabagismo no Brasil a consequência da forte política contra o fumo. ${ }^{22} \mathrm{O}$ segundo aponta que a porcentagem de idosos, que realiza atividade física é 39,52, sendo que dessa quantidade apenas 16,77\% dos entrevistados apresentaram conhecimento adequado sobre o sedenta- rismo e sua relação com as doenças crônicas.

Apesar da informação e o conhecimento não implicarem, necessariamente, em uma mudança comportamental, entende- se que são indispensáveis para que a mudança possa ocorrer e para que as pessoas sigam corretamente os tratamentos de saúde. Por isso se faz necessária a criação de estratégias na promoção da saúde e na educação em saúde do adulto, contemplando as características e os interesses da nossa população idosa, para que, dessa forma, se possa garantir, de fato, que o conhecimento e as ações façam sentido na vida deles, levando em consideração a baixa escolaridade, garantindo o entendimento da informação, primeiro passo para a incorporação do conhecimento.

Este trabalho avaliou uma população de pesquisa homogênea, já que foram abordados idosos com idades, renda, nível de educação e problemas de saúde muito parecidos, moradores de um mesmo bairro; por conseguinte, com um contexto externo semelhante, apesar de a cidade estar localizada na conhecida tríplice fronteira (Argentina, Brasil e Paraguai) e possua um intenso movimento migratório, quase o total de participantes era de brasileiros. Dessa maneira, seria interessante pesquisar grupos mais heterogêneos (estrangeiros, moradores de outros bairros etc.) onde seja possível identificar o conhecimento em grupos distintos.

\section{CONCLUSÕES}

Com o aumento da expectativa de vida da sociedade, aumenta, também, a quantidade de anos com enfermidades. Considerando o contexto social, político e econômico, se faz prioritária para a população e para o sistema de saúde a necessidade de repensar as estratégias de promoção da saúde. Isso porque os resultados descrevem que, apesar das ações desenvolvidas, da difusão na mídia e das campanhas e unidades de saúde, existe ainda desconhecimento sobre os fatores de risco das principais doenças crônicas não transmissíveis.

A importância desse conhecimento reside no impacto positivo que terá na qualidade de vida da população, pois influi na tomada de decisões, seja para a adoção de hábitos saudáveis ou para a realização de tratamentos. É preciso pensar nessas ações, levando em conta as características da população idosa e as características da realidade nacional, para, assim, tentar garantir o entendimento de fato das informações a serem trabalhadas e a apropriação do conhecimento. Mesmo que o conhecimento não implique, necessariamente, na mudança de comportamento é essencial para que possa acontecer ${ }^{25} \mathrm{e}$ melhorar a qualidade de vida. 
Na realização deste estudo, foi percebida a necessidade de um instrumento mais adequado, que permita avaliar o conhecimento de maneira mais apurada, identificando a compreensão real da relação entre os fatores de risco e as doenças. Por esse motivo, sugere-se investir em estudos mais aprofundados na área e, assim, criar ou melhorar instrumentos com as especificidades da população idosa.

Este projeto foi financiado pelo Programa de Bolsas de Iniciação Científica (Probic) da Universidade Federal da Integração Latino-americana. Agradecemos ao mesmo pela oportunidade, assim como à enfermeira Karina Melanésio e aos Agentes Comunitários de Saúde da sua equipe, pela colaboração.

\section{REFERÊNCIAS}

1. Schmidt MI. Chronic non-communicable diseases in Brazil: burden and current challenges. Lancet. 2011; 377(9781):1949-1961.

2. WHO. Global status report on non-communicable diseases 2014. Geneva: World Health Organization, 2014. [Citado 2016 mar. 31]. Disponível em: <http:// www.thelancet.com/journals/lancet/article/PISO140 6736\%2811\%2960135-9/abstract>.

3. WHO. 2008-2013 Action Plan for the Global Strategy for the Prevention and Control of Non-communicable Diseases. 2008. [Citado 2016 mar. 31]. Disponível em: $<$ http://www.who.int/nmh/Actionplan-PC-NCD-2008. pdf $>$.

4. Horton R. GBD 2010: understanding disease, injury, and risk. Lancet. 2012; 380(9859):2053-4.

5. Gaziano TA, Galea G, Reddy KS. Scaling up interventions for chronic disease prevention: the evidence. Lancet 2007; (370):1939-46.

6. IBGE - Instituto Brasileiro de Geografia e Estatística: Pesquisa Nacional de Saúde 2013: percepção do estado de saúde, estilos de vida e doenças crônicas: Brasil, grandes regiões e unidades da federação [Internet]. Rio de Janeiro, Instituto Brasileiro de Geografia e Estatística; [Atualizado em 2014; citado em 2016 mar. 31]. Disponível em: $<$ http://biblioteca.ibge.gov.br/biblioteca-catalogo?view $=$ detalhes\&id $=291110>$.

7. Borges TT. Conhecimento sobre fatores de risco para doenças crônicas: estudo de base populacional [dissertação]. Pelotas: Escola Superior de Educação Física, Uni- versidade Federal de Pelotas; 2008, $130 f$.

8. IBGE - Instituto Brasileiro de Geografia e Estatística: Sinopse do Censo Demográfico 2010 [Internet]. Rio de Janeiro, Instituto Brasileiro de Geografia e Estatística; [Atualizado em 2011 abr.; citado em 2016 mar. 31]. Disponível em: < http://www.censo2010.ibge.gov.br/sinopse $/$ index.php?dados $=12 \& u f=00>$.

9. Chaimowicz F. A saúde dos idosos brasileiros às vésperas do Século XXI: problemas, projeções e alternativas. Rev. Saúde Pública. 1997 abr.; 31(2):184-200.

10. Jardim VCFS, Medeiros BF, Brito AM. Um olhar sobre o processo do envelhecimento: a percepção de idosos sobre a velhice. Rev. Bras de Geriatria e Gerontologia. 2006; 9(2):25-34.

11. Meireles VC, Matsuda LM, Coimbra JAH, Mathias TAF. Características dos idosos em área de abrangência do Programa Saúde da Família na Região Noroeste do Paraná: contribuições para a gestão do cuidado em enfermagem. Rev. Saúde e Sociedade. 2007 jan./abr.; 16(1):69-80.

12. Victor JF, Ximenes LB, Almeida PC, Vasconcelos FF. Perfil sociodemográfico e clínico de idosos atendidos em Unidade Básica de Saúde da Família. Acta Paul Enferm. 2009 ago.; 22(1):49-54.

13. Marin MJS, Castilho NC, Myazato JM, Ribeiro PC, Candido DV. Características dos riscos para quedas entre idosos de uma unidade de saúde da família. Rev. Min. Enf. 2007 out./dez.; 11(4):369-374.

14. Ritcher CM, BetinellI LA, Pasqualotti A, Borges DO, Daltrozo PRO, Klafke JZ, Viecili PRN. Avaliação do conhecimento e da presença de fatores de risco cardiovascular em idosos de Município do Sul do Brasil. Rev. Bras. Cardiol. 2010 set./out.; 23(5):277-285.

15. Anunciação PC, Bragas PG, Almeida PS, Lobo NL, Pessoa MC. Avaliação do conhecimento sobre alimentação antes e após intervenção nutricional entre diabéticos tipo 2. Rev. Baiana de Saúde Pública. 2012 dez.; 36(4):9861001.

16. Domingues MR, Araújo CL, Gigante DP. Conhecimento e percepção sobre exercício físico em uma população adulta urbana do sul do Brasil. Cad. Saúde Pública. 2004 jan./fev.; $20(1): 204-215$. 
17. Borges TT, Rombaldi AJ, Knuth AG, Hallal PC. Conhecimento sobre fatores de risco para doenças crônicas: estudo de base populacional. Cad. Saúde Pública. 2009 jul.; 25(7):1511-1520.

18. Agência Nacional de Saúde Suplementar [Internet]. Promoção da saúde e prevenção de riscos e doenças na saúde suplementar: manual técnico. 2007. [Citado 2016 mar. 31]. Disponível em: <http://bvsms.saude.gov.br/ bvs/publicacoes/promocao_saude_prevencao_riscos_ doencas.pdf $>$.

19. Malcon MC, Menezes AMB, Assunção MCF, Neutzling MB, Hallal PC. Agreement between self-reported smoking and cotinine concentration in adolescents: a validation study in Brazil. J Adolesc Health. 2008 set.; 43(3):226-230.

20. Candeias NMF, Marcondes RS. Diagnóstico em educação em saúde: um modelo para analisar as relações entre atitudes e práticas na área da saúde pública. Rev. Saúde Pública. 1979; (13):63-68.

21. Marinho LAB, Costa-Gurgel MS, Cecatti JG, Osis MJD. Conhecimento, atitude e prática do autoexame das mamas em centros de saúde. Rev. Saúde Pública. 2003 abr.; 37(5):576-582.

22. Freitas MPD. Fatores de risco para doenças cardiovasculares em idosos - coorte de idosos de Bambuí [tese]. Belo Horizonte: Centro de Pesquisas René Rachou, Fundação Oswaldo Cruz; 2011.98f.

23. Cunha M, Chibante R, André S. Social Support, Empowerment and Chronic Disease. Rev Portuguesa de Enfermagem de Saúde Mental. 2014 abr.; (1):21-26.

24. Ramos MP. Apoio social e saúde entre idosos. Rev. Sociologias. 2002 jan./ jun.; (7): 156-175.

25. Knuth AG, Bielemann RM, Silva SG. Conhecimento de adultos sobre o papel da atividade física na prevenção e tratamento de diabetes e hipertensão: estudo de base populacional no Sul do Brasil. Cad. Saúde Pública. 2009 mar.; 25(3):513-520.

Submissão: maio de 2016

Aprovação: abril de 2017 